\title{
Clinical awareness of therapeutic drug monitoring among medical students-A descriptive cross-sectional study
}

\author{
Amberkar Mohanbabu Vittalrao ${ }^{1}$ (D), Aditya Kumar Adhikarla', Sadhana N. Holla ${ }^{1}$ (D), Meena Kumari Kamalkishore ${ }^{*}$ (DD, \\ Seema Kumari Kamal Kishore ${ }^{2}$ \\ ${ }^{1}$ Department of Pharmacology, Kasturba Medical College, Manipal, Manipal Academy of Higher Education, Manipal 576104, Karnataka, India. \\ ${ }^{2}$ Consultant Anaesthesiologist, Mangala Hospital, Hassan, India.
}

\begin{tabular}{l}
\hline ARTICLE INFO \\
\hline Received on: $26 / 05 / 2021$ \\
Accepted on: 22/07/2021 \\
Available Online: 03/11/2021
\end{tabular}

\section{Key words:}

Awareness, curriculum, drug monitoring, pharmacokinetics, regimen.

\begin{abstract}
"A doctor without effective clinical skills is a dodo knowledge." Today's budding medical student is tomorrow's prolific doctor and the pillar of the health care system. The objective was to determine the level of medical students' knowledge on therapeutic drug monitoring (TDM) and create awareness. This study was an institutional-based, descriptive cross-sectional study among the medical students and interns using self-designed, experts validated TDM questionnaire. The questionnaire was divided into Section A - Importance of TDM, Section B - Questions on the core concept, and Section C - On analysis, interpretation, measuring, monitoring, and limitations of TDM. We scored the outcomes for the framed positive and negative questions. On analyzing the grading of scores of students and interns, $11.3 \%$ (44/390) scored excellently, 59.5\% (232/390) showed a good response, and 29.2\% (114/390) reciprocated with a poor grade. We performed an analysis of responses of all three categories related to "most impactful and significant clinical concepts of TDM" requiring in-depth knowledge. The knowledge in medical students, including interns' stands average. The only way to improve this knowledge deficit by conducting well-designed training programs, implementing the concept of TDM so the students are made aware at the undergraduate level and become productive clinically.
\end{abstract}

\section{INTRODUCTION}

"Therapeutic drug monitoring (TDM) is defined as the measurement of a prescribed xenobiotic in serum or biological fluids in a single or multiple time points, to influence prescription and individualize dose regimen to achieve maximal clinical efficacy and minimize adverse effects" (Nwobodo, 2014). The process of TDM is predicated on the assumption that there is a precise relationship between dose, plasma drug concentration, and therapeutic outcome. Therefore, when initiating drug therapy, the physician may find it helpful to measure the plasma drug concentration and tailor individualization of the drug dosage and regimes. A clinical laboratory parameter-TDM requires appropriate

\footnotetext{
${ }^{*}$ Corresponding Author

Meena Kumari Kamalkishore, Department of Pharmacology, Kasturba Medical College, Manipal, Manipal Academy of Higher Education, Manipal 576104 ,Karnataka,India.E-mail: meena.kumari@manipal.edu
}

medical interpretation based on knowledge on pharmaceutics, pharmacokinetics, and pharmacodynamics that can directly influence the drug-prescribing process (Kang and Lee, 2009).

Primarily, TDM is done to track a patient's medication schedule, compliance, and improve outcomes by analyzing drug concentrations. Physiological measures of clinical response, such as lipid concentrations, blood glucose, blood pressure, and clotting tests, are regularly measured by clinicians to track medication pharmacodynamics. However, there is no readily available indicator of effect for specific medications or is inconvenient to obtain. In addition, individualizing medication dosage can be challenging due to significant inter-individual differences in the dose-response relationship. Drugs with small therapeutic indices, wide inter-individual variance in pharmacokinetics parameters, and concentration-dependent pharmacokinetics indicate TDM. In few cases, it is difficult to distinguish between the progress of the disease and the pharmacological effects of a drug. 
Thence, TDM is a critical component in optimizing and clinical management in these cases (Gross, 1998).

"TDM refers to the individualization of drug dosage by maintaining plasma or blood drug concentrations within a targeted therapeutic range or window". Routine monitoring is not advocated for most of the drugs as it is an expensive assay. However, on the other hand, monitoring drug levels is suggested primarily when a specific therapeutic point is difficult to identify. This is not true for all medications, but it is especially essential for those with limited therapeutic ranges, such as lithium, cyclosporine, theophylline, and aminoglycoside antibiotics. For some drugs, TDM helps to assist diagnosis (salicylates), increase efficacy (vancomycin), decrease toxicity (paracetamol), adjust dose requirement (phenytoin, aminoglycosides), assess compliance (anti-epileptics), and finally diagnose under treatment and failed therapy (Sayers and Friedman, 1997).

Drug concentrations must be determined in laboratories with adequately trained personnel and subjected to quality assays within a clinically useful timeframe. The ideal laboratory turnaround time should be shorter than the dosing interval. However, the best sampling time is pre-dose or before a maintenance dose, when a drug with a short half-life is administered by multiple oral doses. For drugs with a long half-life, at least four to five half-lives must elapse before the sample. The analytical methodology employed should ideally be very simple, cost-effective, detect a minimal amount of drug, distinguish between the unchanged drug and its metabolites and remain unaffected by other drugs administered simultaneously. The methods available for analyzing data are highperformance liquid chromatography (HPLC), radio-immunoassay, and enzyme immunoassays. Currently, the most commonly used are fluorescence polarization immunoassay (FPIA) and enzymelinked immunosorbent assay (ELISA), which are specific, precise, and have a short turnaround time (Suthakaran and Adithan, 2006).

TDM needs a team of physicians, nurses, and pharmacists to work together. To ensure that best practices in TDM are accomplished, excellent communication among team members is required. Doctors, pharmacists, and nurses should receive formal instruction in the fundamentals of TDM as a part of their initial training. Continuing education for these practitioners is also expected in hospitals and the general community. The current trend toward greater customer participation in their management indicates that more educational programs are required (Saleem et al., 2020).

"At the student level, it is not the known medical knowledge which is paramount; but utilizing it at the clinical setup in a proper way". TDM is a fundamental tool in the health care sector with no skepticism, and considerable efforts are required to improve the services. Highly recommended ones would be education programs among the clinical team, developing computerbased software, and creating awareness programs among budding healthcare faculties. This brings us to the present study, wherein awareness among the medical students and knowledge on TDM was assessed among Bachelor of Medicine, Bachelor of Surgery (MBBS) students and interns through a questionnaire study.

\section{Aim of study}

To determine the level of knowledge regarding TDM among university MBBS students and interns.
Objectives:

1. To emphasize the importance and clinical awareness among undergraduate students regarding TDM

2. To ascertain their interest in inculcating TDM as a "must do" clinical tool of practice in the future scenario.

\section{MATERIALS AND METHODS}

\section{Ethics approval}

The institutional ethics committee approved the study (Approval no. IEC 248/2015) dated May 13, 2015. The participants were enrolled after the informed consent was obtained.

\section{Design and setting}

This was an institutional-based, observational crosssectional study to assess the awareness of TDM among medical students. It was based on a self-designed, validated questionnaire administered to the study population of undergraduate MBBS students and interns of Kasturba Medical College, Manipal. The study was conducted for 6 months.

\section{Sample size calculation}

The undergraduate medical students from secondyear MBBS to an internship with proper in-depth pharmacology knowledge were included in the study. On the other hand, medical students with a lack of pharmacological exposure toward the concept of TDM like those from First-year MBBS students were excluded from the study. The sample size was calculated by World Health Organization Epi info software (Dean et al., 2013).

Wherein, population size (for finite population correction factor or fpc) $(N): 1,000$, Hypothesized \% frequency of outcome factor in the population $(p): 50 \%+/-5$, Confidence limits as $\%$ of 100 (absolute $+/-\%$ ) (d): 5\%, Design effect (for cluster surveysDEFF):1.

The final calculated sample size for frequency in a $99 \%$ confidence level was 400; however, we did the final assessment only on 390 students.

\section{Data collection}

The survey was conducted with a study tool, a pre-tested, self-administrated questionnaire designed by the investigators after a systematic literature review. Experienced clinicians and professors validated the questionnaire. A well-designed pilot study was conducted among practitioners, ranked scholarly students, and interns for their opinions and modified accordingly.

\section{Study tool}

There were three main sections in the questionnaire:

Section A: had 16 questions on the importance/ awareness of TDM in clinical practice, which had to be answered in the pattern as Yes, No, or Don't know.

Section B: had multiple questions on the core concept of TDM, on which drugs, Why, When, and How to do it? Students had to choose the correct statements.

Section C: Featured questions on analysis, interpretation, measuring, monitoring, and limitations of TDM. The time allocated for the completion of the questionnaire was 30-45 minutes. 


\section{Data analysis}

Entered data and scores were analyzed using Statistical Package for the Social Sciences (SPSS) 16 software (SPSS Inc., Chicago, Ill).

A scoring system measured the outcome according to the framed positive and negative questions.

(A) Positive or negative questions answering correctly: +02 points

(B) Positive or negative questions answering incorrectly: +00 points

(C) If the question has multiple positive or negative answers, each option ticking correctly will carry: +01 points

All these points/scores were converted as percentages, and proportionate frequency in a student population was measured qualitatively by a cross-sectional study. Students' knowledge was analyzed by

1. Grading method as poor $(<40 \%)$, Good $(40 \%-60 \%)$, and Excellent $(>60 \%)$ based on a percentage of correct responses (Figs. 1 and 2).

2. In some of the questions, the stem was common but had multiple answers. It was analyzed by how many percentages of students ticked two, three, four, five, six, and seven correct options (Tables 2 and 4).

3. Some of the framed questions had correct/incorrect Yes, No, and Don't know responses. It was analyzed by a proportion of students ticking these options as correct ones (Tables 3, 5, and Fig. 3).

4. From $\mathrm{A}, \mathrm{B}$, and $\mathrm{C}$ categories, we had chosen questions related to "the most impactful and significant clinical concepts" requiring in-depth knowledge related to the TDM (Fig. 3).

\section{RESULTS}

A total of 390 participants responded comprehensively to the survey. We enrolled 345 MBBS students, among which 178, 94, and 73 students were from the 2nd, 3rd, and 4th year of their MBBS course, respectively, and 45 medical interns participated in our study. On analyzing the grading of scores of students in Figure 1 combining all the three categories of students and interns, $11.3 \%(44 / 390)$ scored excellent response, 59.5\% (232/390) showed a good response, and $29.2 \%(114 / 390)$ reciprocated with a poor grade. Among all respondents, $10 \%$ of second-year MBBS students were graded excellent than $22 \%$ of interns, depicted in Figure 2.

Furthermore, as depicted in Figure 3, we did analyze from all three categories the responses related to "most impactful and significant clinical concepts" requiring in-depth knowledge related to the TDM. In the first category A, we selected four such statements and the first one was negatively framed question "There exists no concept called as "safe drug" or "safe medicine" in therapeutics" (A1) was intelligently answered correctly by $47.9 \%$ of students, and $46.4 \%$ failed to answer correctly. Regarding the statement "TDM is a basic need of current therapeutics because patients'safety becomes the priority" (A6), only $6.15 \%$ of students answered incorrectly. When we asked about the core concept of TDM, "TDM helps in maximizing clinical efficacy and minimizing adverse effects of drugs" (A12), most of them, 90.7\%, gave the correct answer. Finally, a negative question was framed to extract the practical knowledge and awareness of TDM in our hospital "All the clinicians regularly conduct TDM in our hospital" (A13), $27.18 \%$ said YES, which was incorrect, and $38.46 \%$ intelligently ticked NO, which was a correct answer.

In the second category $\mathrm{B}$, regarding "The least fluctuations about drug concentration while dosing is just before the next dose is due" (B3c) statement, only $24.10 \%$ of students answered correctly. From the third category $\mathrm{C}$, we had chosen to state, "The most common body fluid used to measure drug levels is" (C1); a majority of the students, i.e. $84.6 \%$, intelligently ticked "BLOOD" as the correct answer. Finally, regarding the "Drug concentrations should be measured within a clinically useful timeframe in laboratories with appropriately trained staff and subject to quality assays" (C4) statement, 75.90\% of students answered correctly.

\section{DISCUSSION}

This Questionnaire was basically introduced with the idea of stirring up of awareness amongst UG and Interns. When we did the study, it was not there in the regular MCI curriculum. However, now they have introduced competency-based medical education (CBME), and TDM is part of it. Clinical practice is being flooded with drugs. It is tough to find a single branch of clinical therapeutics which is entirely devoid of drug usage. A drug, by default, is poison unless it is used in the optimum dose. There is no concept called "safe medicine" or "safe drug" in therapeutics. That means, "it is a pure lie if a clinician or pharmacist says that the drug is safe." It is paradoxical, especially in the Indian scenario, that a clinician focuses more on drug efficacy, neglecting its adverse or toxic profile. Otherwise, why is there under-reporting of adverse drug effects compared to other developed countries, although we are the second largest populated country having one of the biggest drug consumers? Thence, it is very prudent for a clinician to be vigilant about drug adverse profile, which can be minimized easily by monitoring its serum concentration.

In this study, we had chosen easily understandable and most applied areas of TDM at par with the student's knowledge. From the derived results of the questionnaire study, we found that the overall knowledge and awareness among MBBS students regarding TDM was good. More than half of the students who

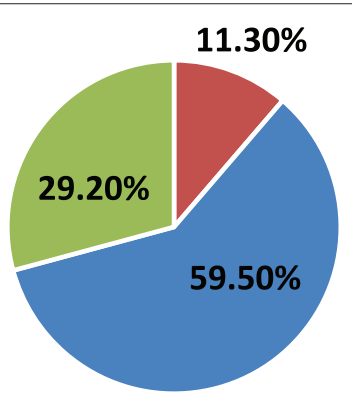

Excellent $\quad$ Good $\quad$ Poor

Figure 1. Grading of scores. 


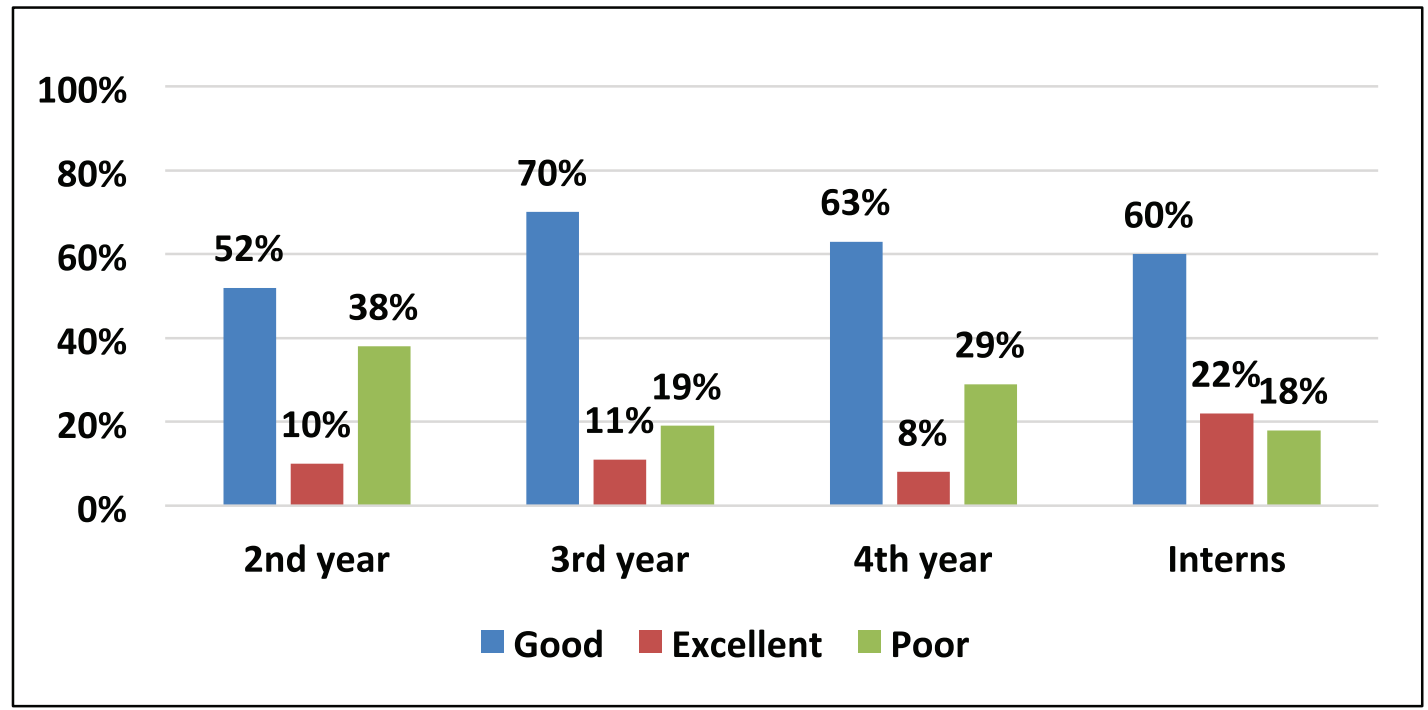

Figure 2. Semester wise grading of scores.

Table 1. Scores of general questions to know the importance of TDM in clinical practice (Category A).

\begin{tabular}{cccc}
\hline Question no & Yes & No & Don't know \\
\hline A1 & $47.9 \%(187)$ & $46.4 \%(181)$ & $5.64 \%(22)$ \\
A2 & $44.62 \%(174)$ & $25.38 \%(99)$ & $30 \%(117)$ \\
A3 & $91.5 \%(357)$ & $5.64 \%(22)$ & $2.82 \%(11)$ \\
A4 & $73.3 \%(286)$ & $12.56 \%(49)$ & $14.1 \%(55)$ \\
A5 & $88.5 \%(345)$ & $10.7 \%(42)$ & $0.8 \%(3)$ \\
A6 & $80.77 \%(314)$ & $6.15 \%(24)$ & $13.08 \%(51)$ \\
A7 & $57.69 \%(225)$ & $14.87 \%(58)$ & $27.44 \%(107)$ \\
A8 & $7.69 \%(30)$ & $88.97 \%(347)$ & $3.33 \%(13)$ \\
A9 & $49.74 \%(194)$ & $29.74 \%(116)$ & $20.51 \%(80)$ \\
A10 & $10.26 \%(40)$ & $80.51 \%(314)$ & $9.23 \%(36)$ \\
A11 & $45.38 \%(177)$ & $52.05 \%(203)$ & $2.56 \%(10)$ \\
A12 & $90.7 \%(354)$ & $8.72 \%(34)$ & $0.51 \%(2)$ \\
A13 & $27.18 \%(106)$ & $38.46 \%(150)$ & $34.36 \%(134)$ \\
A14 & $41.03 \%(160)$ & $54.62 \%(213)$ & $4.36 \%(17)$ \\
A15 & $78.97 \%(308)$ & $7.18 \%(28)$ & $13.85 \%(54)$ \\
A16 & $75.90 \%(296)$ & $11.03 \%(43)$ & $13.08 \%(51)$ \\
\hline
\end{tabular}

Table 2. Multiple correct answer type of questions for the concept of TDM: which drugs, why, when and how to do it? (Category B).

\begin{tabular}{ccccccc}
\hline $\begin{array}{c}\text { Question } \\
\text { Number }\end{array}$ & Wrong Answer & One Correct Answer & Two Correct Answers & $\begin{array}{c}\text { Three Correct } \\
\text { Answers }\end{array}$ & $\begin{array}{c}\text { Four Correct } \\
\text { Answers }\end{array}$ & $\begin{array}{c}\text { Five Correct } \\
\text { Answers }\end{array}$ \\
\hline B1 & $9 \%(35)$ & $28.3 \%(110))$ & $29.5 \%(115))$ & $24.6 \%(96))$ & $8.7 \%(34)$ & - \\
B2 & $7.9 \%(31)$ & $15.6 \%(61)$ & $25.6 \%(100)$ & $30 \%(117)$ & $14.4 \%(56)$ & $6.4 \%(25)$ \\
B4 & $11.8 \%(46)$ & $21.3 \%(83)$ & $13.1 \%(51)$ & $17.2 \%(67)$ & $13.8 \%(54)$ & $22.8 \%(89)$ \\
B3b & $14.1 \%(55)$ & $73.3 \%(286)$ & $12.56 \%(49)$ & - & - \\
B3d & $64.6 \%(252)$ & $27.9 \%(109)$ & $6.7 \%(26)$ & - & $0.7 \%(3)$ & - \\
\hline
\end{tabular}


Table 3. Scores of yes, no and don't know questions for the concept of TDM (Category B).

\begin{tabular}{cccc}
\hline Question number & Yes & No & Don't know \\
\hline B3a & $76.15 \%(297)$ & $23.08 \%(90)$ & $0.77 \%(3)$ \\
B3c & $24.10 \%(94)$ & $67.69 \%(264)$ & $8.21 \%(32)$ \\
B3e & $62.82 \%(245)$ & $15.13 \%(59)$ & $22.05 \%(86)$ \\
B5 & $60.51 \%(236)$ & $12.05 \%(47)$ & $27.44 \%(107)$ \\
\hline
\end{tabular}

Table 4. Multiple correct answer type of questions for TDM analysis, interpretation, measuring, monitoring and limitations.

\begin{tabular}{ccccccccc}
\hline $\begin{array}{c}\text { Question } \\
\text { number }\end{array}$ & $\begin{array}{c}\text { Wrong } \\
\text { answer }\end{array}$ & $\begin{array}{c}\text { One correct } \\
\text { answer }\end{array}$ & $\begin{array}{c}\text { Two correct } \\
\text { answer }\end{array}$ & $\begin{array}{c}\text { Three } \\
\text { correct } \\
\text { answer }\end{array}$ & $\begin{array}{c}\text { Four correct } \\
\text { answer }\end{array}$ & $\begin{array}{c}\text { Five correct } \\
\text { answer }\end{array}$ & $\begin{array}{c}\text { Six correct } \\
\text { answer }\end{array}$ & $\begin{array}{c}\text { Seven } \\
\text { correct } \\
\text { answer }\end{array}$ \\
\hline $\mathrm{C} 3$ & $19.2 \%(75)$ & $27.4 \%(107)$ & $22.8 \%(89)$ & $14.9 \%(58)$ & $8.5 \%(33)$ & $2.8 \%(11)$ & $0.8 \%(3)$ & $3.6 \%(14)$ \\
$\mathrm{C} 7$ & $7.7 \%(30)$ & $9.7 \%(38)$ & $14.4 \%(56)$ & $35.6 \%(139)$ & $15.1 \%(59)$ & $17.4 \%(68)$ & & \\
$\mathrm{C} 1$ & $15.3 \%(60)$ & $84.6 \%(330)$ & & & & & \\
\hline
\end{tabular}

Table 5. Scores of true, false and don't know questions for TDM analysis, interpretation, measuring, monitoring and limitations.

\begin{tabular}{cccc}
\hline Question number & True & False & Don't know \\
\hline C2 & $71.28 \%(278)$ & $26.41 \%(103)$ & $2.31 \%(9)$ \\
C4 & $75.90 \%(296)$ & $21.03 \%(82)$ & $3.08 \%(12)$ \\
C5 & $70 \%(273)$ & $25.13 \%(98)$ & $4.87 \%(19)$ \\
C6 & $63.85 \%(249)$ & $14.87 \%(58)$ & $21.28 \%(83)$ \\
\hline
\end{tabular}

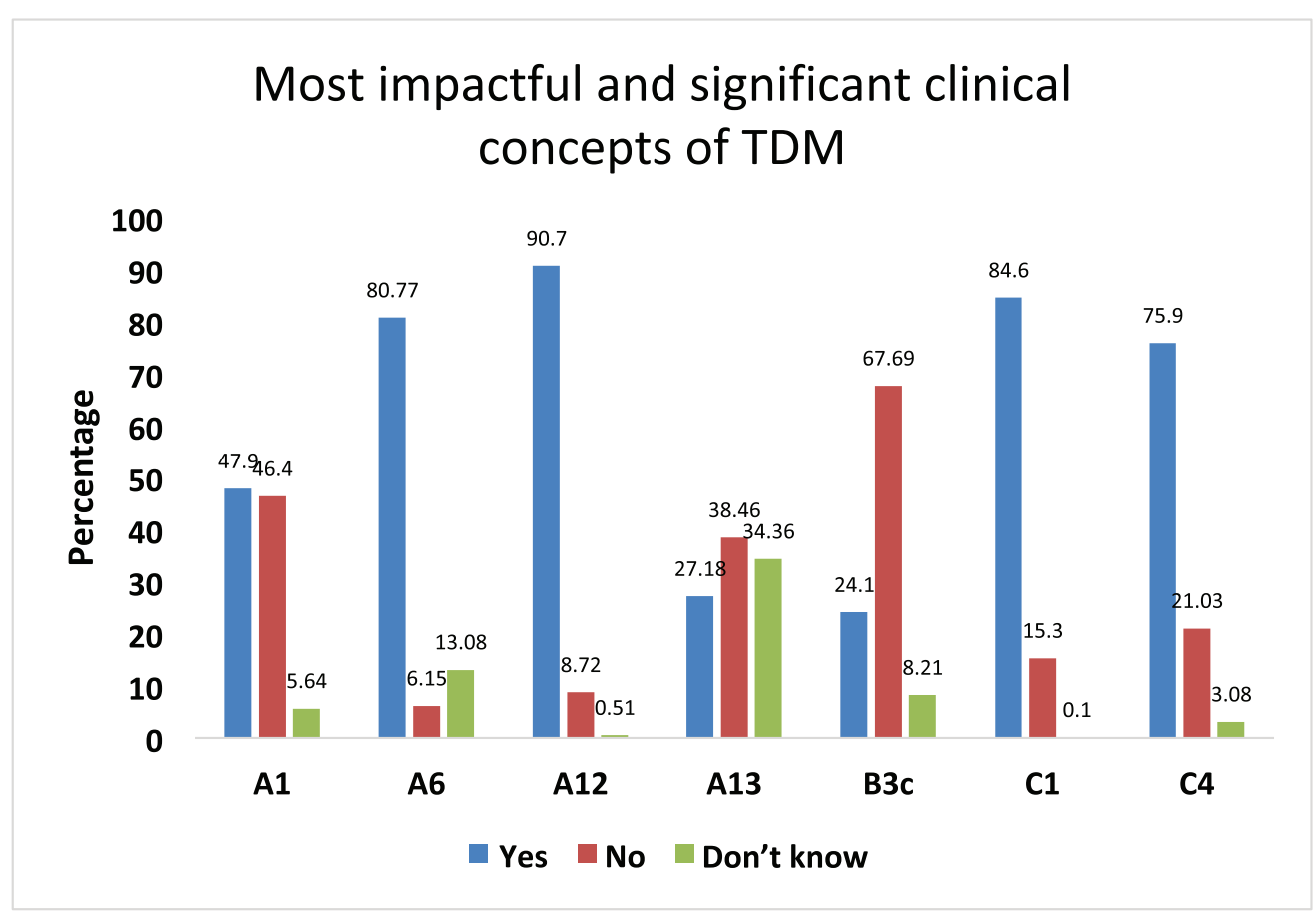

Figure 3. Analysis of most impactful and significant clinical concepts of TDM. 
took part in the survey answered correctly to all TDM questions (Fig. 1). In a study conducted on Australian medical students to investigate their knowledge and skills in pharmacology, $63.3 \%$ of them had a piece of adequate knowledge on TDM (Bullock and Leversha, 2019).

The level of knowledge regarding TDM was relatively increasing from the second year to interns. As per grading, the good responses were seen in $70 \%$ of third-year MBBS, $63 \%$ of fourth-year MBBS students, and $60 \%$ of interns compared to $52 \%$ responses of 2nd-year MBBS students (Fig. 2). Thus, the level of perception of clinical pharmacology knowledge and skills was better across the years of course. A study conducted by Latha et al. (2015). on knowledge and awareness of TDM showed a significant difference in second and final-year MBBS students. The secondyear students were better in terms of knowing what happens to a drug in the body. In contrast, the final year students were better at understanding the clinical aspects of TDM.

The internship is a crucial period between undergraduation and clinical practice. Over the period, the knowledge and experience in clinical pharmacology are better. It was evident that $22 \%$ of interns' responses were excellent compared to the second year MBBS students which was $10 \%$ (Fig. 2). A crosssectional study conducted on interns concluded that clinical pharmacology topics like TDM, dosage calculation, adverse drug reaction reporting, paediatric and emergency medicine needed more weightage in their undergraduate teaching curriculum (Nitya et al., 2013).

However, there is a significant knowledge gap among MBBS students, including interns, regarding theoretical and practical knowledge of TDM. It could be due to the old curriculum, which does not cover the clinical considerations of TDM. In comparison to application-oriented knowledge (i.e., applying a drug treatment), undergraduate medical students were more optimistic in their pharmacology knowledge (i.e., drug indications) (Johannsen et al., 2019). Interestingly, a study reported that the pharmacy students have shown to have significantly better knowledge of drug interactions compared to medical students, which suggests that this may be due to differences in inculcating and implementing TDM as a part of undergraduate academic education between these two health professions (Keijsers et al., 2014).

On analyzing the general questions of TDM in clinical practice, many of the students did believe that TDM defines a close relationship between plasma level of drug and clinical effect. TDM as a clinical tool gives a greater insight into patients' drug therapy, maximizing clinical efficacy and minimizing adverse effects, and prioritizes patients' safety (Table 1 and A12). To understand the value of TDM for practicing physicians, a hypothesis was considered way back in 1989 based on the assumption that there is a well-defined target range where the patient develops maximum clinical benefit and minimum toxicity. Dose adjustment in this range decreases inter-individual variations (McInnes, 1989). Recent technical advancements have sparked renewed interest in personalized medicine. Standard technology areas such as clinical drug monitoring, on the other hand, have remained underappreciated. Throughout observation and implementation of TDM in clinical practices, the drug monitoring services and analyzing the pharmacokinetic data resulted in fewer adverse reactions, shorter intensive care, and decreased overall stay in the hospital (Gawade, 2016).

The knowledge of students on the cost and feasibility of TDM were assessed. Although the students were aware of the beneficial effects of TDM, they found it could be time-consuming to the clinicians; around $80 \%$ of them reciprocated it this way. Half of them felt that the TDM procedure could burden the patient because it is of wastage of human resources, manpower, and money. Students were also aware that TDM is an expensive practice compared to routine investigations. The majority felt that it could be advised to only those who are affordable (Table 1 and A15). Recent studies reflect that the most significant benefit of TDM is for a set of populations such as neonates, elderly age, impaired renal function, immunosuppressed, patients on anticancer chemotherapy, psychopharmacological, and anticonvulsant drugs. In these situations, safe and humane practice considers TDM a necessary clinical procedure in optimizing evidencebased medicine without being cost-conscious. Owing to the present clinical scenarios, TDM could improve patient outcomes positively and reduce overall healthcare costs (Ates et al., 2020; Schumacherand and Barr, 2002).

Around $44.62 \%$ of the students answering the questionnaire knew the status of TDM practice in our hospital, and the remaining $55.38 \%$ of them were not aware of the TDM practice (Category A2). However, $57.69 \%$ of the students were ready to incorporate TDM as part and parcel of their clinical practice in the future (Category A7). The interest of students and interns in clinical pharmacology displayed an exclusive need of incorporating the TDM syllabus in the theory and practical curriculum of medical undergraduates, and $45.38 \%$ of them agree with this thought (Table 2, Category A11). Throughout the different phases of development of curriculum for clinical pharmacology for medical undergraduates, it is shown that there was an absolute need to implement these pharmacokinetic profiles of a drug as basic skills at the beginning of undergraduate clinical training, learn its application during the internship and clinical practice (Gitanjali and Shashindran, 2006). Increasing the understanding of the basic pharmacokinetic concepts related to TDM led to the development of e-learning tools. This tool was found most helpful in health professionals who performed TDM on a day-to-day basis. Such simulated computer-based self-directed e-learning exercises can be made available for students (Samani, 2009).

TDM entails not only determining drug concentrations but also interpreting the findings clinically. It necessitates a thorough understanding of pharmacokinetics, sample time, drug history, and the patient's clinical condition. On analyzing the core concept of TDM for which drugs, why, when, and how to do it, the students were provided with multiple questions about appropriate indications and drugs that necessitate plasma monitoring, around only $29.5 \%$ of the students were able to pick at least two correct answers in the questionnaire. Only $8.7 \%$ of the total answered could select all the four accurate indications for TDM (Table 2, B1). The indications are poorly defined clinical endpoints, preventing adverse drug effects, sizeable inter-individual variability, and compliance. Deliberately, incorrect distractors were included as options, e.g., TDM is the unnecessary procedure for drugs with a wide therapeutic range, TDM as a routine investigation, and 
the need for TDM when the pharmacological effects could be clinically quantified (Table 2, B1 and B2).

Current guidelines on TDM help the clinician make an appropriate clinical decision, but it is not used as a routine laboratory test. It can help assess patient response to therapy and distinguish between non-compliant and true non-responders. In antibiotic therapy failure, TDM helps to differentiate between inadequate plasma concentration or bacterial resistance. TDM can also detect drug toxicity or adverse effects before clinical signs appear, which may save lives. Increasing trough gentamicin concentrations, for example, could signal possible renal damage early. TDM is a test that can be used to establish a drug interaction that has been suspected. Co-administration of an enzyme inducer or inhibitor, for example, is likely to alter cyclosporine's pharmacokinetics, reducing its effectiveness. Patients who have impaired clearance of a drug like renal failure have decreased clearance of medications like digoxin which has a narrow therapeutic index. Providing TDM of digoxin in such patients will help clinicians adjust dose regimens accordingly (Ali et al, 2013). In this study, the students had a fair knowledge of the drugs, which requires strict TDM. Among drugs, like lithium, cyclosporine, digoxin, gentamicin, and sodium valproate, $30 \%$ chose three correct answers, and $6.4 \%$ of them got all the five right (Category B2 and Table 2).

Awareness and knowledge of sampling times for TDM were evaluated among the students and interns. To know when to do the procedure requires basic knowledge of pharmacokinetic principles of drug monitoring. Blood samples are usually collected at a steady-state to obtain serum drug concentration. When a fixed dosage is given at regular intervals, the drug builds up in the body until the rate of drug absorption equals the rate of drug removal, known as the steady-state. The time it takes to reach a steady state is determined by the drug's elimination half-life. A onecompartment distribution model takes 4-5 half-lives for first-order kinetics to reach a steady state (Schumacher, 1985). In this study, $76.15 \%$ of them could rightly define steady-state concentration, and $73.3 \%$ knew that steady-state concentration could be reached after four plasma $t_{1 / 2}$ (Category B3a and B3b, Tables 2 and 3). The appropriate time for taking a TDM sample is immediately before the next dose when the drug concentration is low-trough concentration. Sometimes, a sample collected immediately after drug administration at a peak concentration may be required. Evaluation of the test result's clinical significance depends on the knowledge of the time when the sample was drawn. Among the students, only $24.10 \%$ knew that the least variable point regarding drug concentration while dosing is just before the next dose is due, whereas the majority of the $67.69 \%$ got it wrong and $8.21 \%$ were unaware about it (Category B3c, Table 3).

To summarize, plasma samples for TDM are generally taken after two plasma $t_{1 / 2}$, while samples for drugs with long halflives, such as phenobarbitone, may be taken at any point during the dosing interval. In some instances, sampling may detect toxicity at the time of specific symptoms due to peak concentrations, such as in lithium. For once-daily drug administration, the blood sample is taken post-dose or at peak concentration (Ghiculescu, 2008). The majority of them could not interpret the options (64.6\%), only $27.9 \%$ could identify one right answer (Category B3d, Table 2). So, it is essential to educate the students on the importance of sampling time with respect to steady-state concentration and the last dose. Abnormal or unpredictable results will be produced if sampling is done incorrectly. To prevent misinterpretation of findings, physicians and the team should always be aware of proper sampling times (Ali et al, 2013).

At three Malaysian hospitals, the appropriateness of sampling times and indications for monitoring serum drug concentrations for TDM were assessed. Overall, the findings from the three hospitals were promising, with nearly $80 \%$ of requests being deemed correctly indicated. Still, the percentage of appropriateness sampling time was lower at $30 \%$, indicating that more work was required (Hamzah and Rahman, 2008).

"Drug assay" is requested for TDM for documentation purposes, including the timing of the sample collection, the time of the last dose, dosage regimen, clinical indication, and concomitant medication with comorbid conditions. In this section, most of the students got five correct answers (22.8\%), and the rest could at least get few answers in the correct way (Category B4, Table 2). Age, body weight, time of first and last dose, dosing interval, the route of administration, information on the other co-administered drugs, and sampling times must be accurately documented in the patient's medical records and the TDM form. According to the "Consensus Guidelines" for TDM, a request form with all the above elements is needed for optimal TDM practice, without which interpretation is impossible (Salek et al., 2020).

In most drug assays, total drug concentration (bound and unbound drug) is measured. Still, only the unbound drug interacts with its receptor/target site to produce a response; $62.82 \%$ of the students could answer this right (Category B3e, Table 3). Factors like serum albumin concentration, displacement reaction, and renal dysfunction influence the unbound fraction. This concept is paramount for drugs like phenytoin, which has $>90 \%$ plasma protein binding capacity and follows dose-dependent kinetics. Suppose the unbound fraction of phenytoin doubles from $10 \%$ to $20 \%$, the target range based on total phenytoin concentration should be halved. Phenytoin toxicity will occur if dosage changes are not made according to the normal target range. So, it is essential to know the usual therapeutic range of drugs like phenytoin (10 $20 \mathrm{microgram} / \mathrm{ml}$ ), sodium valproate (10-20 microgram $/ \mathrm{ml})$, carbamazepine (5-10 microgram $/ \mathrm{ml})$ and lithium $(0.5-0.8 \mathrm{mEq} / \mathrm{l})$ at the undergraduate MBBS student level. Students were unable to recognize that the therapeutic range for phenytoin is not $50-100$ microgram $/ \mathrm{ml}$ during the evaluation of this section. The majority, $60.51 \%$ were incorrect (Category B5, Table 3), suggesting the need to classify applied aspects of the target therapeutic range of clinically relevant drugs. However, to guide the clinicians, reference ranges for commonly monitored drugs are available in many TDM handbooks (Ali et al, 2013).

The measurement of drug assays is crucial for the interpretation and analysis of TDM. The most common body fluid used to measure drug levels is blood. Many of the $84.6 \%$ of them got it right (Category C1, Table 4). As there could be variation in pharmacokinetics and pharmacodynamics for few drugs hindering plasma drug assessment, urine and saliva can also be used for analysis. As a result, the dosage, route of administration, pattern of drug usage, and dispositional kinetics (distribution, metabolism, and excretion) of the drug all determine drug concentrations in biological fluids (Chiang and Hawks, 1986). The knowledge of students on different methods to measure drug assays was 
analyzed. From the options of different tools given, only $8.5 \%$ of them got it right (Category C3, Table 4). Analytical methods ranging from radioimmunoassay to HPLC procedures were used by drug testing laboratories to develop their assay procedures. HPLC is currently the most commonly used tool, but there are also FPIAs, enzyme immunoassays, and ELISAs (Nitin et al., 2019).

TDM is a very effective tool, and research has shown that it can be much more helpful when used in conjunction with proper guidelines. The benefits of therapeutic monitoring of drugs and their usefulness, in the long run, were assessed. Out of all, $71.28 \%$ felt that TDM helps in judging the clinical outcome of under or overtreatment (Category C2, Table 5). The majority of them, $75.9 \%$, felt that drug concentrations should be measured within a clinically helpful timeframe in laboratories with appropriately trained staff and subject to quality assays (C4, Table 5). It is essential that the laboratory turnaround time should be shorter than the dosing interval depending upon the feasibility. Among all, 63.8\% of them approved that drug monitoring and interpretive services may help to improve the safety, efficacy, and cost-effectiveness of clinical services (Category C6, Table 5). Finally, $70 \%$ of them had an opinion that TDM could be used as an educational aid by promoting the principles of rational prescribing and quality use of medicines, which was positive feedback. (Category C5, Table 5).

Apart from the limited number of drugs amenable to TDM, there are also few drawbacks. Among the limitations, $35.6 \%$ could get at least three right options, and $17.4 \%$ got all the five options correct. The limitations of TDM were discussed. It was based on the scientific accuracy and precision of the drug assays, laboratory variability in reporting, documenting, limited accessibility, and lack of infrastructure, especially in the developing countries like India. The further limitations were the differences between laboratories' validated target ranges, lack of commitment, knowledge, and awareness about TDM among doctors (Category C7, Table 4). TDM's theoretical foundation is well-established. Many practical aspects of TDM, however, restrict the utility of drug monitoring (Walson, 1985).

\section{Study limitations}

We concede the fact that 1 . When students randomly replied to our survey (390 out of 1,000), we may have overlooked a large number of scholarly students. It could have served as a deterrent to students' awareness of being labelled as average 2. The study would have been more comprehensive if working clinicians/ senior residents/clinical pharmacologists/pharmacists were also made as participants. When contrasted to the understanding of MBBS students, this could have resulted in the disparity in TDM awareness 3. Even though the students were extremely sensitive to our questionnaire, we could not rule out the possibility of them having difficulty focusing and answering correctly. This laid-back attitude may have resulted from the long hours of exhaustive teaching classes.

\section{CONCLUSION}

The knowledge deficit assessed could have been minimized by inculcating the "must do" or "must learn" TDM as a clinical tool in both practical/theory MBBS curricula. TDM contributes to the advancement of safe and appropriate therapeutic drugs. Unfortunately, TDM awareness among medical students, including interns, is average; these major flaws may be addressed by implementing several well-designed clinical training programs to be well-versed in these topics beginning in graduate school. Each student must be aware of TDM. This is made possible only if the concept of TDM is taught with utmost seriousness having a practical approach like more problem-based learning, discussions on case scenarios, live case demonstrations, and e-learning modules. With the introduction of the CBME curriculum for the graduates in second-year MBBS, TDM is made a mandatory component in the course curriculum and must make efforts to train the undergraduate students from the theory basics to the clinical application of TDM.

\section{ACKNOWLEDGMENTS}

The authors would like to thank all MBBS medical students from second, third, fourth year, and Interns for their utmost operation and reciprocating with their clarity of knowledge.

\section{LIST OF ABBREVIATION}

ADR Adverse drug reaction

CBME Competency based medical education

ELISA Enzyme-linked immunosorbant assay

TDM Therapeutic drug monitoring

FPIA Fluorescence polarization immunoassay

HPLC High-performance liquid chromatography

MBBS Bachelor of Medicine, Bachelor of Surgery

SPSS Statistical Package for the Social Sciences

\section{AVAILABILITY OF DATA AND MATERIALS}

Study data and other related materials are available with authors.

\section{AUTHORS' CONTRIBUTIONS}

All the authors contributed to the study in a prolific way. Dr. Amberkar Mohanbabu Vittalrao did conceptualize the study design, acquisition, analysis, and interpretation of data. Dr. Aditya Kumar Adhikarla did material preparation, data collection, and statistical analysis. Dr. Amberkar Mohanbabu Vittalraoscripted the first draft of the manuscript, Dr. Aditya Kumar Adhikarla, Dr. Sadhana N Holla, Dr. Meena Kumari K, and Dr. Seema Kumari K revised it critically for important intellectual content. Dr. Meena Kumari $\mathrm{K}$ has reviewed and commented on the manuscript. Finally, all authors reviewed and approved the final manuscript to be published.

\section{ETHICS APPROVAL}

The study was conducted in compliance with ethical standards. The institutional ethics committee approved the study (Approval no. IEC 248/2015).

\section{CONFLICT OF INTEREST}

The authors declare that they have no conflict of interest. 


\section{FUNDING}

This project was awarded Indian Council Medical Research-Short term studentship F.N0.21/1/2015-BMS-STS.

\section{REFERENCES}

Ali AS, Abdel-Rhaman MS, Rahman AF, Osman OH. Basic principles of therapeutic drug monitoring. J Appl Biopharma Pharmacokinet, $2013 ; 1: 87-95$.

Ates HC, Roberts JA, Lipman J, Cass AEG, Urban GA, Dincer C. On-site therapeutic drug monitoring. Trends Biotechnol, 2020; 38(11):1262-77.

Bullock S, Leversha A. Medical student perceptions of their knowledge and skills in pharmacology in their first and final clinical years. MedEdPublish, 2019; 8(1);42.

Chiang CN, Hawks RL. Implications of drug levels in body fluids: basic concepts. NIDA Res Monogr, 1986; 73:62-83.

Dean AG, Sullivan KM, Soe MM. OpenEpi: open source epidemiologic statistics for public health, version. ScienceOpen, Inc., Berlin, Germany, 2013. Available via www.OpenEpi.com (Accessed 0509 2015).

Gawade SP. Overview on monitoring of therapeutic drugs. IJOPP, 2016; 9(3):152-6.

Ghiculescu RA. Therapeutic drug monitoring: which drugs, why, when and how to do it. Aust Prescr, 2008; 31:42-4.

Gitanjali B, Shashindran CH. Curriculum in clinical pharmacology for medical undergraduates of India. Indian J Pharmacol, 2006; 38(Suppl 2): S108-14.

Gross AS. Best practice in therapeutic drug monitoring. Br J Clin Pharmacol, 1998; 46:95-9.

Hamzah A, Rahman ABF. Evaluation of blood sampling times and indications for therapeutic drug monitoring services. Malays J Pharm Sci, 2008; 6(1):1-11.

Johannsen W, Frings B, Herzig S, Matthes J. Development of perceived pharmacological deficits of medical students and alumni supports claim for continuous and more application-oriented education. Naunyn Schmiedeberg's Arch Pharmacol, 2019; 392:29-36.

Kang JS, Lee MH. Overview of therapeutic drug monitoring. Korean J Intern Med, 2009; 24(1):1-10.

Keijsers CJ, Brouwers JR, de Wildt DJ, Custers EJ, en Cate OT, Hazen AC, et al. A comparison of medical and pharmacy students knowledge and skills of pharmacology and pharmacotherapy. Br J Clin Pharmacol, 2014; 78(4):781-8.

Latha RA, Rajeswaramma G, Kumari DA, Srinivasu K. A questionnaire based study on knowledge and awareness of therapeutic drug monitoring (TDM) among 2nd, 3rd and final year medical students of govt. medical college, Ananthapuramu. AP. India. J Evol Med Dent Sci, 2015; $4(4): 630-6$
McInnes GT. The value of therapeutic drug monitoring to the practising physician-an hypothesis in need of testing. Br J Clin Pharmacol, 1989; 27(3):281-4.

Nitin BC, Flavia CZ, Nikhil A, Ramesh B, Tambuwala, MM, Clementi E. Bio-analytical assay methods used in therapeutic drug monitoring of antiretroviral drugs-a review. Curr Drug Ther, 2019; 14(1):16-57.

Nitya S, Mangaiarkkarasi A, Meher Ali R, Sawadkar Maruthi Sripathi. Intern's knowledge of clinical pharmacology and therapeutics at Puducherry: a cross-sectional study. Int J Basic Clin Pharmacol, 2013; 2(5):622-8

Nwobodo N. Therapeutic drug monitoring in a developing nation: a clinical guide. JRSM Open, 2014; 5(8):2054270414531121.

Saleem MA, Basharat R, Rana NA, Khattak SA. Role of clinician in therapeutic drug monitoring practice. Clinic Pract, 2020; 17(1):1429-35.

Salek T, Schneiderka P, Studena B, Votroubkova M. Survey on request form content and result reporting in therapeutic drug monitoring service among laboratories in Czechia and Slovakia. Biochem Med (Zagreb), 2020; 30(2):020706

Samani K. Therapeutic drug monitoring: an e-learning resource. Biosci Horiz, 2009; 2(2):113-24.

Sayers J, Friedman M. How clinicians use therapeutic drug monitoring. Lab Med, 1997; 28(8):524-8.

Schumacher GE. Choosing optimal sampling times for therapeutic drug monitoring. Clin Pharm, 1985; 4(1):84-92.

Schumacherand GE, Barr JT. Therapeutic drug monitoring: is it cost-effective? Exp Rev Pharmacoecon Outcome Res, 2002; 2(6):619-24.

Suthakaran C, Adithan C. Therapeutic drug monitoringconcepts, methodology, clinical applications and limitations. Health Administrator, 2006; 19(1):22-6.

Walson PD. Practical problems of therapeutic drug monitoring of anticonvulsants. Brain Dev, 1985; 7(2):116-21.

How to cite this article:

Vittalrao AM, Adhikarla AK, Holla SN, Kamalkishore MK, Kishore SKK. Clinical awareness of therapeutic drug monitoring among medical students-A descriptive crosssectional study. J Appl Pharm Sci, 2021; 11(11):034-045. 


\section{SUPPLEMENT FILE}

\section{TO EVALUATE THE CLINICAL AWARENESS OF THERAPEUTIC DRUG MONITORING (TDM) AMONG MEDICAL UNIVERSITY STUDENTS}

Name and Course:

Date:

\section{A. GENERAL QUESTIONS TO KNOW THE IMPORTANCE OF TDM IN CLINICAL PRACTICE:}

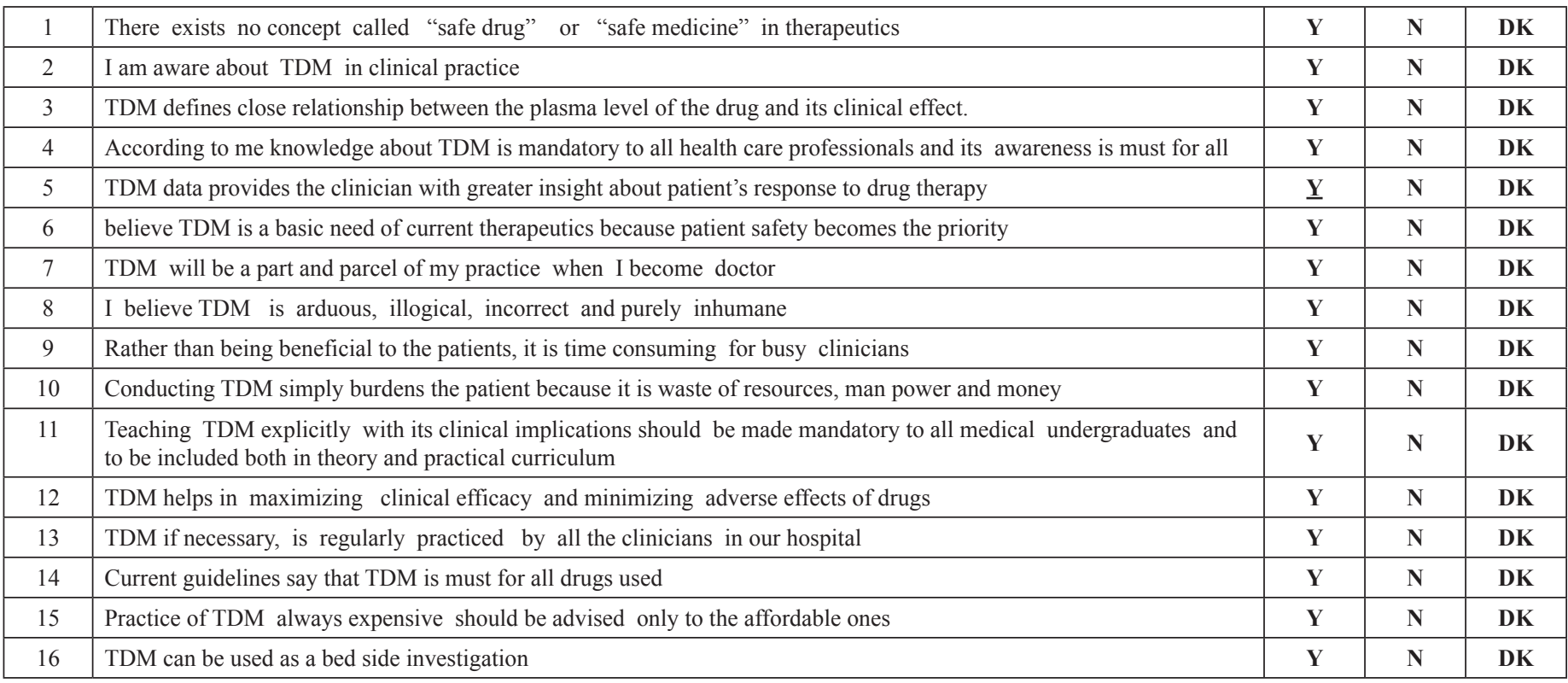


KINDLY NOTE: 1) THIS STUDY INCLUDES BOTH CORRECT AND INCORRECT STATEMENTS

2) FEW QUESTIONS CARRY MULTIPLE ANSWERS TICK THE CORRECT CHOICES IF U KNOW OTHERWISE LEAVE IT BLANK

\section{B. THE CONCEPT OF TDM: WHICH DRUGS, WHY, WHEN AND HOW TO DO IT:}

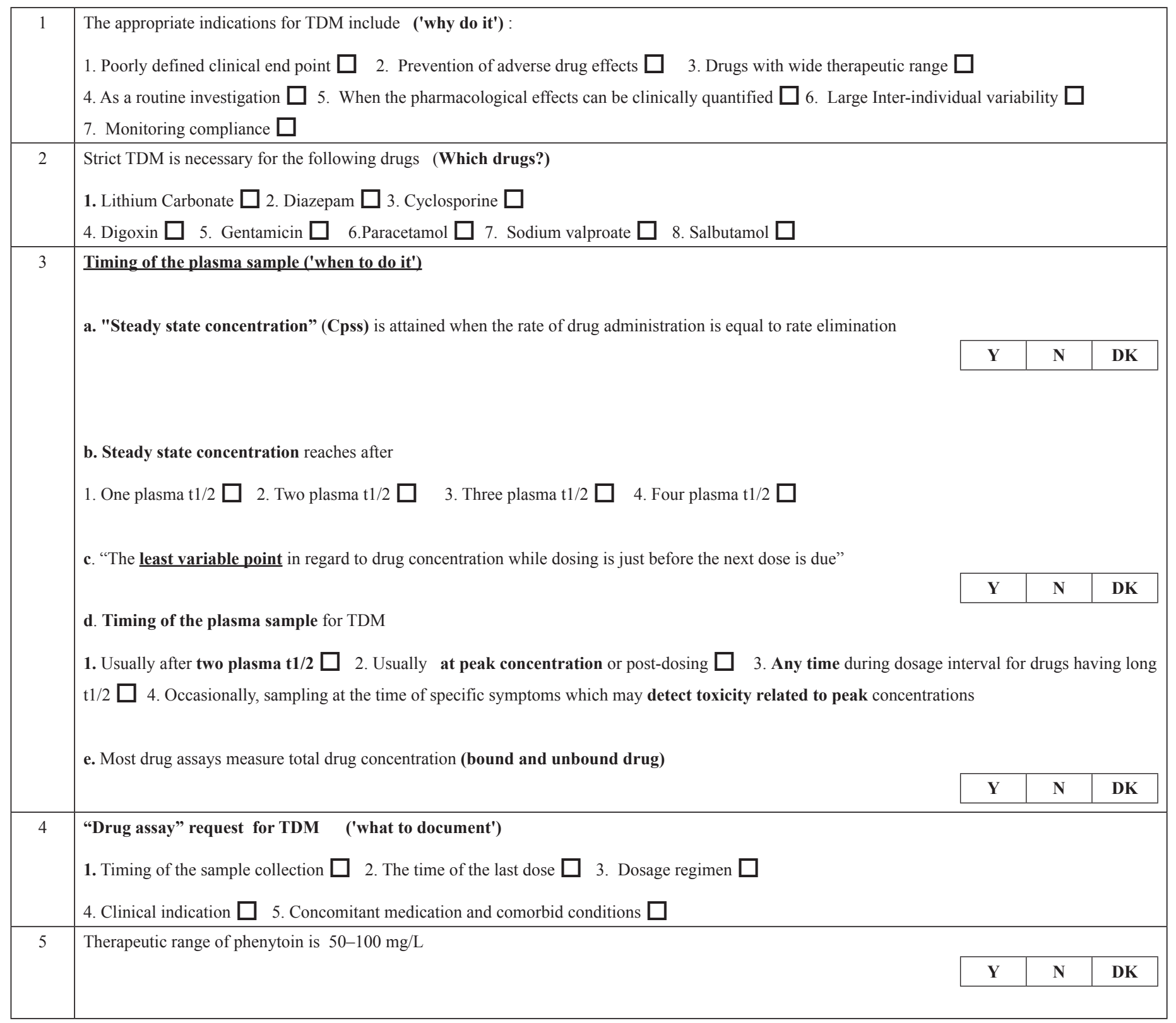




\section{TDM ANALYSIS, INTERPRETATION, MEASURING, MONITORING AND LIMITATIONS}

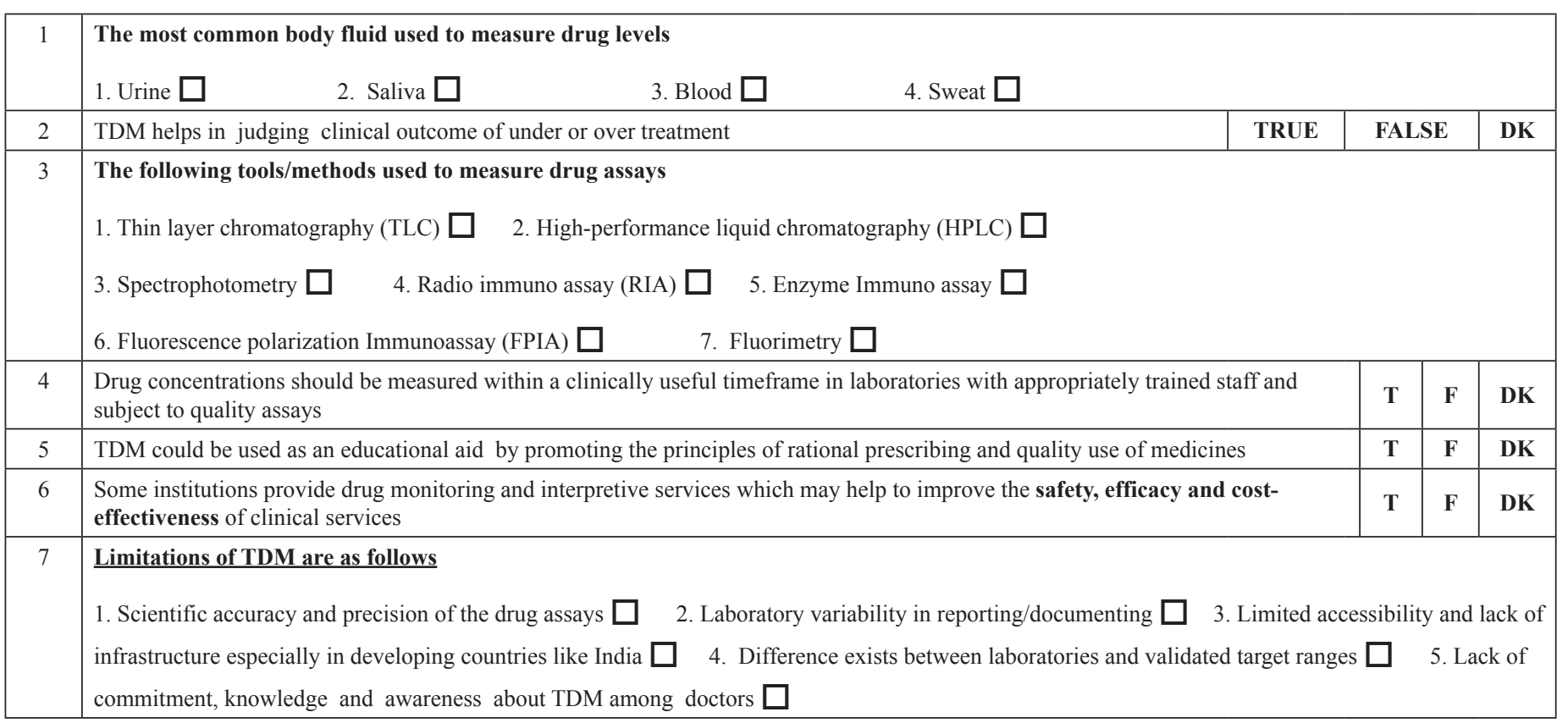

